\title{
Realizing Lobe-Specific Aerosol Targeting in a 3D-Printed In Vitro Lung Model
}

\author{
Emily L. Kolewe, BS, ${ }^{1, *}$ Yu Feng, $\mathrm{PhD}{ }^{2}$ and Catherine A. Fromen, $\mathrm{PhD}^{1, *}$
}

\begin{abstract}
Background: Delivery of aerosols to isolated lobes of the lungs would be beneficial for diseases that have lobespecific effects, such as cancer, pneumonia, and chronic obstructive pulmonary disorder. Recent computational fluid-particle dynamic (CFPD) modeling has demonstrated that in low flow rates, the inlet location of a particle at the mouth dictates the lobe into which it will deposit. However, realization of this lobe-specific deposition has yet to be attempted experimentally or in the clinic. To address this, we sought to develop a proof-of-concept in vitro model and targeting device for achieving lobe-specific delivery.

Methods: Using 3D printing, a lung replica was created from a computed tomography scan of a healthy 47year-old male volunteer and connected to a flow setup to control inlet flow rate and outlet airflow distribution to each lobe. A device was designed and fabricated that directs particles to an inlet location that is $5 \%$ of the total inlet area and described by radial coordinates $(r, \theta)$. Filter paper at sampling ports for each lobe was used to capture fluorescent polystyrene particles to quantify particle collection. We evaluated lobe-specific targeting at varied inlet coordinates, particle diameters, inlet flow rates, and disease lobe flow rate distribution profiles.

Results: Guided by CFPD modeling, inlet locations were identified that increased particle collection to a target lobe between $63 \%$ and $90 \%$. For example, release of fluorescent particles at the inlet location $r=4.67 \mathrm{~mm}$, $\theta=252^{\circ}$ with respect to the center of the inlet using $1 \mu \mathrm{m}$ particles, $1 \mathrm{~L} / \mathrm{min}$ inlet flow rate, and healthy subject lobe flow distribution profile yielded $90 \%$ of the aerosol dose to the right upper lobe, corresponding to an increase of $4.6 \times$ above the non-targeted percent particle collection. Particle size, inlet flow rate, and disease airflow distributions were all shown to generally decrease the efficiency of lobe-specific targeting.

Conclusions: Our results indicate that aerosol targeting of a specific lobe is possible in vitro under optimized conditions and that controlling inlet locations could be a potentially useful method for treatment of lobe-specific diseases. This is the first demonstration of lobe-specific particle collection in a physical lung model and illuminates numerous challenges that will be faced as this method is translated to clinical applications.
\end{abstract}

Keywords: 3D printing, device development, in vitro/in silico model, lobe specific, lobe targeting, targeting

\section{Introduction}

I NHALED THERAPEUTICS ARE WIDELY CONSIDERED BENEFICIAL for respiratory conditions, as this method of delivery can transport therapeutics directly to the affected site. ${ }^{(1)}$ For patients with diseases such as chronic obstructive pulmonary disorder (COPD), pneumonia, and lung cancer, pathology occurs non-uniformly in the airspace, leading to lobe-specific regional effects. ${ }^{(2-4)}$ However, airways in these diseased regions often do not receive adequate inhaled ther- apeutics. ${ }^{(5)}$ When mucus, bacteria, constrictions, or tumors block airways, airflow can be diverted away from diseased tissue and directed toward healthy regions, carrying the therapeutic with it. ${ }^{(5-8)}$ As a result, many respiratory diseases, including lung cancer and pneumonia, are more commonly treated with intravenous (IV) and/or oral therapeutics. Using inhalable therapeutics in place of oral or IV would reduce systemic effects and improve delivery to the disease site, which has led to clinical trials for inhalable cancer therapeutics such as doxorubicin. ${ }^{(5,9)}$ However, undesirable

\footnotetext{
${ }^{1}$ Department of Chemical and Biomolecular Engineering, University of Delaware, Newark, Delaware, USA.

${ }^{2}$ School of Chemical Engineering, Oklahoma State University, Stillwater, Oklahoma, USA.

*Member of ISAM.
} 
off-target effects to the healthy areas of the lungs remain detrimental to the development of such inhalable therapeutics. There is a need to improve disease targeting of aerosols within the lung to reduce off-target effects and lower the barrier for developing novel inhalable therapeutics.

Historically, attempts to target differential regions of the lungs have been met with some noteworthy successes. Most commonly, differential regions of the lung can be accessed by manipulating particle aerodynamic diameter to control the generation of delivery. ${ }^{(10,11)}$ This is useful for diseases such as asthma, which generally affects the conducting airways of all lobes evenly, or for targeted delivery to the alveoli for systemic transport. ${ }^{(12,13)}$ However, the symptoms of COPD, pneumonia, and cancer are often localized to a lung or lobe and would benefit from targeting an individual lobe in addition to a generation. ${ }^{(2-4,11)}$ Segment-specific targeting has been demonstrated in limited scenarios, resulting in controlled delivery to differential regions of the lung. In preclinical studies, magnetic particles have been employed to direct distribution to either the right or left lung of mice. ${ }^{(14)}$ Disease state and posture were shown to innately influence the lobe of delivery in rats. ${ }^{(15)}$ Clinically, patient posture has been shown to direct distribution to axial, medial, and basal lung regions in human patients ${ }^{(16)}$ and liquid release from a bronchoscope has been used to locally direct delivery toward a tumor. ${ }^{(17)}$ These studies support the general concept that regional and even lobe-specific targeting is not only desirable but also may be achievable in relevant preclinical and clinical environments.

Further, recent computational fluid-particle dynamic (CFPD) modeling has suggested that it is possible to control the lobe of delivery by using the particle inlet location and patient-specific streamlines. ${ }^{(18-20)}$ An overview diagram of this concept is shown in Figure 1. Although highly promising for lobe-specific targeting, such controlled aerosol release into the lung has yet to be physically evaluated in either preclinical or clinical studies. Previous left or right lung delivery has been assessed by using mouse models to evaluate biological responses ${ }^{(14)}$; here, we are interested in using a model that more accurately mimics the geometry of human lungs and can assess lobe-level aerosol distribution, rather than particle-size distributions obtained via an impactor. $^{(21)}$ In the past decade, researchers turned to $3 \mathrm{D}$ printing replicas of lungs derived from computed tomography (CT) scans for quantification of aerosol distribution. ${ }^{(11)}$ Idealized mouth-throat and upper airway models have been used to compare against known in vivo data and improve in vitro-in vivo correlations. ${ }^{(22,23)}$ In addition, researchers have printed patient replicas out to several generations and tracked particles to better understand the effects of geometry

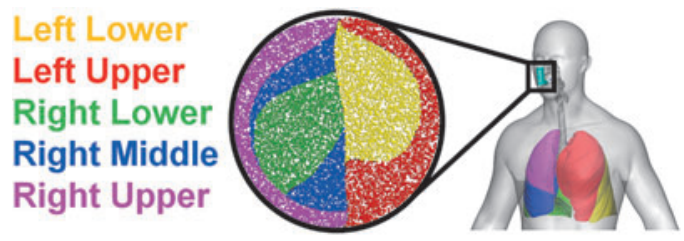

FIG. 1. Regional targeting diagram. CFPD modeling showing the inlet particle map of release position that is color-coded to the lobe of deposition. CFPD, computational fluid-particle dynamic. on aerosol trajectory. ${ }^{(24,25)}$ The 3D-printed models of human airways expand the scope of quantifiable data in aerosol research, especially in the absence of clinical studies, allowing for detailed evaluation of particle deposition and offering potential for the assessment of regional aerosol delivery.

We endeavored to create a lung model and aerosol device to demonstrate the feasibility of lobe-specific deposition by using controlled inlet locations. Guided by CFPD predictions, we created a device to control aerosol inlet locations and evaluated its efficacy in a corresponding 3D-printed lung replica with lobe-outlet flow rates controlled through valves, flow meters, and a vacuum pump. Using this approach, we demonstrate individual targeting of each of the five human lung lobes, with percent particle collection increasing to the target lobe by 55\%-95\%. This corresponds to a two-fold to five-fold increase in percent particle collection over the untargeted controls and is highly dependent on the particle size, inlet flow rate, and the lobe-level airflow distribution. Through the following experiments, we evaluate conditions to promote aerosol lobe-specific targeting and provide experimental evidence toward the potential for such personalized delivery approaches that could create future opportunities for novel devices and therapeutics in the lungs.

\section{Materials and Methods}

\section{CFPD: Geometries}

CFPD was performed to generate particle release maps (see Fig. 1 as an example) that indicate particle release positions at the inlet associated with their lung deposition sites, whether that inlet be the mouth opening or a midtrachea position. Specifically, the Euler-Lagrange method enhanced with in-house $C$ programs ${ }^{(26)}$ was employed to simulate airflow and both particle transport and deposition in two airway configurations. The geometry of the human respiratory system geometry was built from previous studies, with the complete geometry of a healthy 47-year-old male subject from mouth to generation 6 (G6) and a mouth opening simplified as a circular disk with $20 \mathrm{~mm}$ in diameter, as a representative cross-section. ${ }^{(26)}$ A second, truncated airway configuration was also considered to match experiments, and it includes the trachea to main bronchi (described next in the Lung Replica section). Airflow was considered as fully developed Poiseuille flow at the trachea inlet, based on the fact that the length of the straight tube upstream to the trachea inlet is sufficiently long for the airflow to become fully developed. Computational approaches initially considered particle release with constant velocity uniformly across the inlet, where the particle velocity at the inlet is equal to the average velocity of the airflow. Complete and simplified regional targeting devices (RTDs) with inner tubes at different locations were then evaluated within the geometries to investigate the device effects on the disturbance of pulmonary airflows and inhaled particle trajectories and fates.

\section{CFPD: Governing equations}

Pulmonary airflow dynamics is always unsteady and driven by the pressure differences under the action of the cyclic breathing process. The flow field in the airways was 
assumed to be incompressible. The conservation laws of mass and momentum can be written in tensor forms as follows:

$$
\begin{gathered}
\frac{\partial\left(\rho u_{j}\right)}{\partial x_{j}}=0 \\
\frac{\partial u_{i}}{\partial t}+u_{j} \frac{\partial u_{i}}{\partial x_{j}}=-\frac{1}{\rho} \frac{\partial p}{\partial x_{i}}+\frac{1}{\rho} \frac{\partial \tau_{i j}}{\partial x_{j}}+g_{i},
\end{gathered}
$$

where $u_{j}$ represents the fluid velocity, $p$ is the pressure, $g_{i}=(9.81,0,0)\left[\mathrm{m} / \mathrm{s}^{2}\right]$ is gravity, and viscous stress tensor $\tau i_{j}$ in Equation (2) is given by:

$$
\tau_{i j}=\mu\left[\left(\frac{\partial u_{i}}{\partial x_{j}}+\frac{\partial u_{j}}{\partial x_{i}}\right)-\frac{2}{3} \delta_{i j} \frac{\partial u_{k}}{\partial x_{k}}\right] .
$$

The shear stress transport transition model ${ }^{(27,28)}$ was adapted in this study, based on its overall excellent performance, predicting "laminar-to-turbulent" transition onset, computational efficiency, and reasonable accuracy when compared with large eddy simulation.

A Lagrangian frame of reference was employed to track each particle. Assuming aerosol particles to be spheres, in light of the large particle-to-air density ratio, and negligible thermophoretic forces, the reduced particle trajectory equation reads:

$$
\frac{d}{d t}\left(m_{p} u_{i}^{p}\right)=F_{i}^{D}+F_{i}^{L}+F_{i}^{B \mathrm{M}}+F_{i}^{G} .
$$

Here, $u_{i}^{p}$ and $m_{p}$ are the velocity and mass of the particle, respectively; $F_{i}^{D}$ represents the drag force, ${ }^{(29)} F_{i}^{G}$ is the gravity, ${ }^{(30)} F_{i}^{B M}$ is the Brownian motion-induced force, and $F_{i}^{L}$ is the Saffman lift force. ${ }^{(31)}$

\section{CFPD: Parameters and assumptions}

ANSYS CFX and Fluent 2019 R (ANSYS, Inc., Canonsburg, PA, USA) were employed for the CFPD simulations. Particle diameter $d_{p}$ was varied from 1 to $10 \mu \mathrm{m}$, and the particle density $\rho_{p}$ was consistently $1000 \mathrm{~kg} / \mathrm{m}^{3}$. The inhalation flow rate $Q_{\text {in }}$ was varied from 1 to $10 \mathrm{~L} / \mathrm{min}$. Numerical simulations were performed on a local 64-bit Dell Precision T7910 workstation with 128 GB RAM and sixteen $3.1 \mathrm{GHz}$ CPUs and the supercomputers in the High Performance Computing Center at Oklahoma State University (e.g., Cowboy cluster machine with 252 standards compute nodes with dual Intel Xeon E5-2620 "Sandy Bridge" hex core $2.0 \mathrm{GHz}$ CPUs, with $32 \mathrm{~GB}$ of $1333 \mathrm{MHz}$ RAM). The boundary conditions used can be summarized as follows:

1. Realistic flow rate distributions were employed among five lobes to reflect the healthy and diseased lung conditions. $^{(32)}$

2. A random-parabolic particle distribution was used at the inlet, where the random particle positions were generated from an in-house MATLAB code.

3. Particle-wall interaction boundary condition was assumed to be a " $100 \%$ trapped wall," meaning the airway will capture particles at initial contact.
Computational meshes were generated by using the commercial software ANSYS Fluent Meshing 2019 R. A multi-layer region consisting of dense hybrid polyhedral/pentahedral elements was generated near the wall surface to contain the viscous sub-layers fully and to resolve any geometric features present there.

\section{$3 D$ printing}

All parts were printed on a Carbon M1 3D printer (Carbon, Inc., Redwood City, CA, USA), cleaned, and postmodified according to the manufacturer's recommendations. Three proprietary resins were used, namely elastic polyurethane (EPU) 40, prototyping resin (PR) 25 White, and rigid polyurethane (RPU) 60. For parts printed in PR, parts were cleaned with isopropyl alcohol (IPA) before being exposed to ultraviolet light to cure remaining resin for 15-second intervals until all areas were exposed and parts were no longer tacky. For parts printed in EPU and RPU, parts were cleaned briefly with IPA for 1 or 5 minutes, respectively, before being baked to cure remaining resin for 8 or 4 hours, respectively. When using the Carbon M1 3D printer, our printer settings utilized the dynamic light synthesis setting.

\section{Regional targeting device}

An RTD was designed to align with the inlet of a lung model, such that an inner tube $5 \%$ of the total inlet area directs the location where fluorescent particles enter the model and is surrounded by an outer tube that houses nonfluorescent particles (Fig. 2A, B). Non-fluorescent particles were used to replicate the CFPD simulation conditions as closely as possible, where particles uniformly enter the lung model. The inner tube location controls the fluorescent particle location and, thus, the streamlines that the particles will follow through the model. The targeted lobe of particle collection can be controlled by changing the inlet location (Fig. 2C, D). The RTD was designed in Solidworks and printed by using the Carbon M1 3D Printer. Parts were printed, cleaned, and postmodified according to the manufacturer's recommendations described earlier.

The CFPD modeling is color-coordinated with each lobe (Fig. 1 and 2D), and it is used to predict which locations would be best to target with an RTD. Four RTDs were created with the inner tube at specific radii from the center of the outer tube, $r=2.7,4.7,6.2$, and $7.7 \mathrm{~mm}$ (Supplementary Fig. S1). A peg was added on the outer wall of the RTD that aligns with notches in the lung model at rotation angles of $\theta=36^{\circ}, 108^{\circ}, 180^{\circ}, 252^{\circ}$, and $324^{\circ}$. Between potential radial and angular coordinates, there are 20 potential locations that would be available for targeting (Fig. 2C, D) and are replicated by using the notches in the lung replica and peg in the four RTDs. For convention, all inlet locations are reported as radius (in $\mathrm{mm}$ ) and angle (in degrees).

\section{Lung replica}

A lung replica was generated to align with the RTD and connect to the in vitro flow apparatus according to Figure 3. The 3D-rendered lung of a healthy male 47-year-old subject developed for CFPD modeling ${ }^{(26)}$ was given a $2 \mathrm{~mm}$ wall thickness (Supplementary Fig. S2A) by using Autodesk 

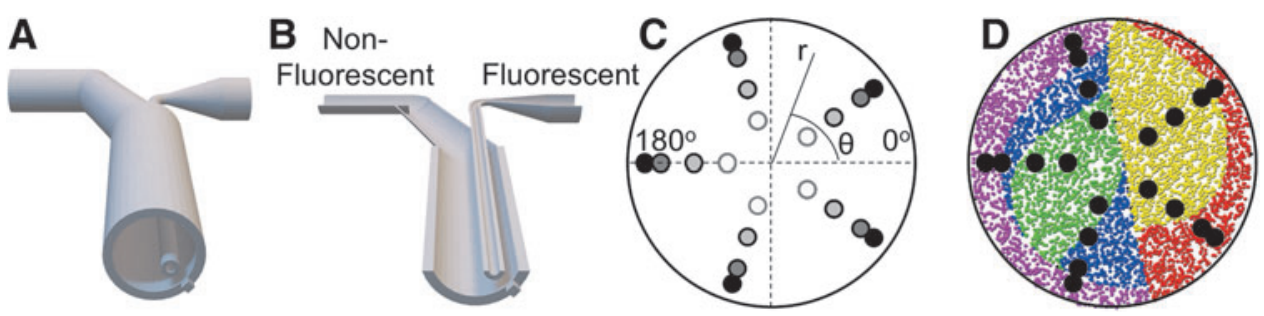

FIG. 2. RTD design. RTD full device (A) and cross-sectional view (B) showing two outlets that connect to a non-fluorescent and fluorescent aerosol reservoir and the inner tube, which directs the fluorescent particle inlet location. (C) The map of possible inlet locations based on the radii $(r=2.67,4.67,6.17$, and $7.67 \mathrm{~mm})$ of RTDs and the angles $\left(\theta=36^{\circ}, 108^{\circ}\right.$, $180^{\circ}, 252^{\circ}$, and $324^{\circ}$ ) achievable with the peg-notch combinations used in this study. (D) The alignment of these possible locations with the CFPD predicted inlet map where the colors are coordinated to each lobe: left lower (yellow), left upper (red), right lower (green), right middle (blue), and right upper (purple). RTD, regional targeting device.

Meshmixer (San Rafael, CA, USA). This was then manipulated in Solidworks (Waltham, MA, USA) such that the bronchi below generation 2 or 3 were removed, leaving an outlet to each lobe, and the outlets were extended (Supplementary Fig. S2B). Another lung model was created by truncating the model at the upper airway from the glottis

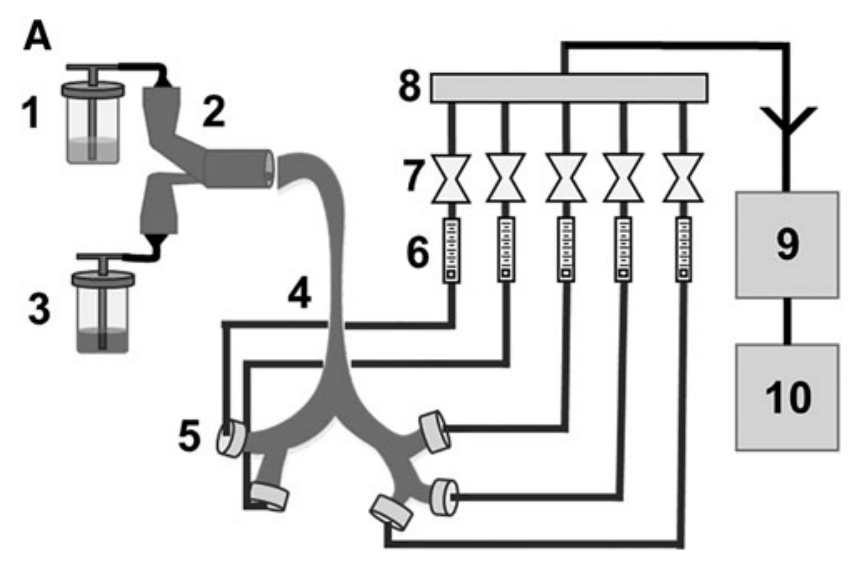

B

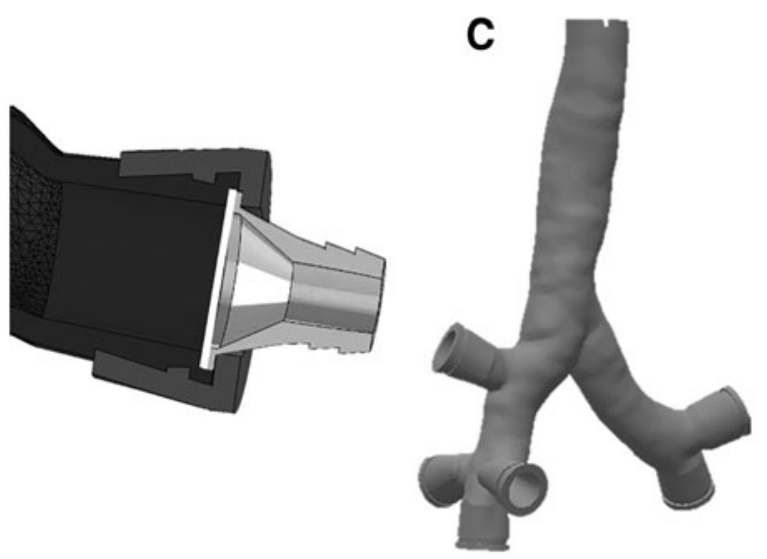

FIG. 3. Experimental setup. (A) Schematic of the control system used to test RTD. (1) Non-fluorescent particle CJN, (2) RTD, (3) fluorescent particle CJN, (4) lung replica, (5) lobe sampling cap, (6) flow meters, (7) valves, (8) manifold, (9) critical flow controller, and (10) vacuum pump. (B) Sliced view showing the lung model (black), lobe sampling cap (dark gray), filter paper placement (white), and barbed tubing connection (light gray). (C) The intubated lung model. CJN, collision jet nebulizers. onward (Supplementary Fig. S2C) to neglect the turbulent region of the glottis. In intubation, a patient has a tube inserted through the mouth, passed the glottis, and into the trachea, essentially bypassing the upper throat. Thus, the truncated model is referred to as the "intubated model," intended to mimic the release position of an aerosol in an intubated patient (Fig. 3C). Notches around the inlet were added to ensure inlet locations, and device alignment was reproducible (Supplementary Fig. S2E). These were then printed by using the Carbon M1 3D Printer to generate a lung replica (Supplementary Fig. S2F). The lung replica was printed in PR with a modulus of $2000 \mathrm{MPa}$. Elastic EPU caps and PR hard, barbed tubing connections were designed to hold filter paper in place against the model (Supplementary Fig. S3) and also printed on the Carbon M1 3D Printer. Parts were printed, cleaned, and postmodified according to the manufacturer's recommendations as described earlier.

\section{Experimental setup}

Flow meters, valves, tubing, and barbed tubing connections (McMaster Carr, Elmhurst, IL, USA) connected each lobe outlet to a manifold according to the diagram in Figure 3A. The manifold combined the flow streams to one stream and connected to the Critical Flow Controller and vacuum pump (MSP, Shoreview, MN, USA). Flow meters (Product ID 41695K32; McMaster Carr) had a range of 1$280 \mathrm{~mL} / \mathrm{min}$ and thus were replaced with an anemometer (Series 0560 1405; Testo 405i Anemometer, Testo) when performing at a flow rate of $10 \mathrm{~L} / \mathrm{min}$; they were connected via a 3D-printed barbed tubing connection (Supplementary Fig. S10). Collison jet nebulizers ( $\mathrm{CH}$ Technologies, Westwood, NJ, USA) were filled with $30 \mathrm{~mL}$ methanol and $100 \mu \mathrm{L}$ Fluoresbrite YG fluorescent or non-fluorescent polystyrene particles (Polysciences, Warrington, PA, USA). The particles exiting the RTD were monodisperse and the aerodynamic diameter was quantified by using a nextgeneration impactor (MSP), shown in Supplementary Figure S4. Calculations were performed to confirm that methanol adequately evaporated before particles entered the lung model (see Supplementary Data, Supplementary Fig. S5, and Supplementary Table S1). Adapters were 3D printed in EPU by using the Carbon M1 3D Printer to connect the nebulizers to the RTD and the RTD to the lung 
model. Our experimental setup was intentionally grounded to avoid electrostatic buildup and designed to enable independent airflow control at the outlet of each lobe. The overall flow rate was measured at the model inlet, whereas each lobe outlet set point was established based on airflow distributions reported for reference "healthy" subjects by Sul et al. (Table 1; higher flow rates in Supplementary Table S2). ${ }^{(32)}$ Additional experiments were run by adjusting the lobe outlet flow rate distribution to match those reported of a patient with $\mathrm{COPD}^{(32)}$ and establishing an intermediate profile between the two distributions (Table 1). To validate the model at each outlet condition, the percent particle collection distribution of fluorescent particles uniformly entering into the lung replica was compared with the airflow distribution.

Polystyrene $1 \mu \mathrm{m}$ particles were used with an inlet flow rate of $1 \mathrm{~L} / \mathrm{min}$ unless otherwise indicated. Fluorescent particles were released solely from the inner tube of the RTD, whereas non-fluorescent particles were released from the surrounding region. Particles of larger diameter (up to $10 \mu \mathrm{m}$ ) were explored for CFPD modeling, and particles of 2.65 and $7.55 \mu \mathrm{m}$ diameter were used in the in vitro model based on the CFPD findings. This range was chosen due to a combination of pharmaceutically relevant small particles and larger particles being known to deposit in the upper airways, such as those considered in our model. ${ }^{(1)}$ In general, low flow rates were evaluated to ensure laminar flow and avoid mixing due to turbulence. The low flow rate of $1 \mathrm{~L} / \mathrm{min}$ was chosen based on CFPD guidance and previous results, ${ }^{(18,19)}$ whereas the higher flow rate of $10 \mathrm{~L} / \mathrm{min}$ was chosen to approach more physiologically relevant flow rates. For the geometry used and standard experimental conditions of $1 \mu \mathrm{m}$ particles and $1 \mathrm{~L} / \mathrm{min}$, the inlet Reynolds number and Stokes number are 94.2 and $1.47 \mathrm{e}-5$, respectively (see Supplementary Data for calculations).

Particles were "inhaled" in the model for 10 seconds and collected on $10 \mu \mathrm{m}$ cellulose filter paper (Fisher Scientific, Waltham, MA, USA) at the end of each lobe. Filter paper of pore size $10 \mu \mathrm{m}$ was used to ensure adequate air flow at the outlets of the model and to prevent vacuum buildup that is present with filter paper of smaller pore sizes. We estimate filter collection efficiency to be equivalent for all lobe out-

Table 1. Model Airflow Distributions

\begin{tabular}{|c|c|c|c|c|c|c|}
\hline \multirow[b]{2}{*}{ Lobe } & \multicolumn{2}{|c|}{ Healthy } & \multicolumn{2}{|c|}{ Intermediate } & \multicolumn{2}{|c|}{$C O P D$} \\
\hline & Percent & $\begin{array}{c}\text { Flow } \\
\text { rate } \\
\text { (L/min) }\end{array}$ & Percent & $\begin{array}{c}\text { Flow } \\
\text { rate } \\
\text { (L/min) }\end{array}$ & Percent & $\begin{array}{c}\text { Flow } \\
\text { rate } \\
(\mathrm{L} / \mathrm{min})\end{array}$ \\
\hline LL & 23.7 & 0.237 & 17.0 & 0.170 & 10.0 & 0.100 \\
\hline LU & 23.7 & 0.237 & 26.0 & 0.260 & 29.0 & 0.290 \\
\hline RL & 18.7 & 0.187 & 16.0 & 0.160 & 13.0 & 0.130 \\
\hline RM & 14.0 & 0.140 & 10.0 & 0.100 & 5.0 & 0.050 \\
\hline RU & 20.3 & 0.203 & 31.0 & 0.310 & 43.0 & 0.430 \\
\hline
\end{tabular}

Outlet flow rate settings for each lobe used in the in vitro model shown as percent per lobe and measured outlet rate per lobe at a mouth inlet flow rate of $1 \mathrm{~L} / \mathrm{min}$. Outlet distributions based on clinically relevant flow rate percent per lobe reported for a healthy subject and patient with COPD in Ref. 32. An intermediate model condition was established by averaging the LL distribution between the two reported clinical distributions.

COPD, chronic obstructive pulmonary disorder; LL, left lower; LU, left upper; RL, right lower; RM, right middle; RU, right upper. lets between $70 \%$ and $98 \%$ (see Supplementary Data for calculations) and assume that the particles collected on each filter were representative of the total particles entering that lobe. After collection, filter paper from each lobe was removed and imaged by using a Biotek Gen5 Cytation (Biotek, Winooski, VT, USA) with excitation $441 \mathrm{~nm}$ and emission $485 \mathrm{~nm}$. Five representative images were taken for each filter paper. Particles in each image were counted by using Image (NIH, Bethesda, MD, USA) and averaged for the five representative images of each lobe filter. The lobe-specific percent of particles collected was calculated as the ratio of the number of counted particles on each lobe filter paper to the total number of all particles counted on all six filters; that is, the sum of particles collected across all filters. Notably, the right middle (RM) lobe had two outlets due to patient-specific geometry. Because of this, the particle deposition on those filters was summed together and divided by two to account for normalizing to the area of the other lobe outlets.

\section{Statistical analysis}

All samples were performed in triplicate, and results were reported as an average and standard deviation. Each experiment resulted in a targeted collection profile, which was compared against the corresponding non-targeted collection profile by using a two-way analysis of variance (ANOVA) that was used in GraphPad Prism 8 (San Diego, CA, USA). For example, when using position $\left(r=6 \mathrm{~mm}, \theta=36^{\circ}\right)$ and inlet flow rate of $10 \mathrm{~L} / \mathrm{min}$ with $1 \mu \mathrm{m}$ diameter particles in the intubated model, the resultant collection profile was compared against non-targeted data of no device (and thus no position referenced), inlet flow rate of $10 \mathrm{~L} / \mathrm{min}, 1 \mu \mathrm{m}$ diameter particles, and the intubated model. In addition, to quantify the increase in percent particle collection only in the desired targeted lobe, a Student's $T$-test was used in Microsoft Excel (Redmond, WA, USA) assuming two-tailed distribution and equal variance. Additional Student's $T$-tests were also used to quantify decreases in percent particle collection between lobes after targeted and non-targeted particle collection. Resulting $p$-values are represented with symbols for $p<0.05(*), p<0.01(* *), p<0.001(* * *)$, and $p<0.0001(* * * *)$.

\section{Results}

\section{Model validation}

To validate the experimental setup, the percent collection of particles was expected to match the percent flow rate to each lobe. For example, because the left lower (LL) lobe received $23 \%$ of the airflow, it was expected to receive $23 \%$ of the particles that are collected in the entire model. This comparison was performed for both the full throat and intubated lung models. In our experimental setup, there was no statistical difference between the airflow and the particle collection (Fig. 4), indicating that the model accurately reflected the clinically relevant geometry, flow rates, and collection profiles. This also demonstrated that the particles collected were representative of the particles entering the model, as was previously hypothesized. Notably, no collected particles were detected on the 3D-printed lung replica. This demonstrates that particle interactions, such as electrostatic surface interactions, are negligible in our model. 


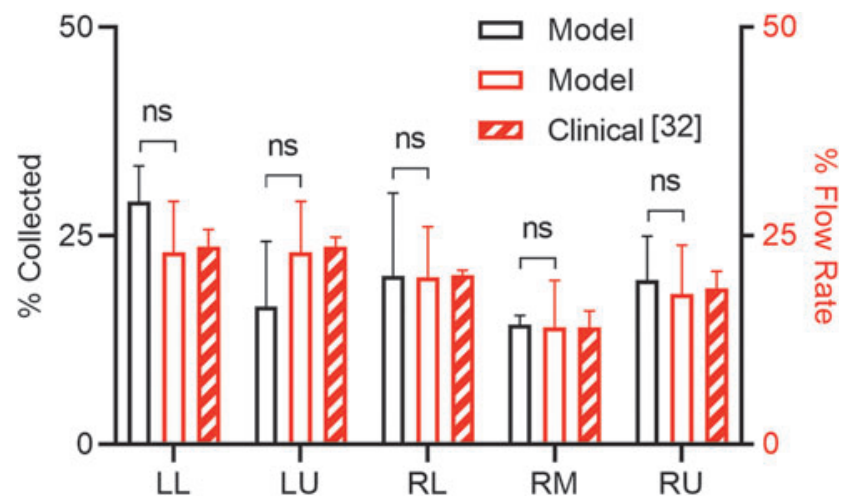

FIG. 4. Model validation. Comparison of reference clinical airflow distributions (red slash), ${ }^{(32)}$ achieved experimental airflow rate distribution (red solid), and the percent particle collection (black open) for each lobe outlet. Clinical reference values were obtained from reported healthy subject and COPD patient data collected by Sul et al. ${ }^{(32)}$ Statistical analysis was performed by using two-way ANOVA, $n=3, p=0.278$ and 0.99 , respectively, between the achieved airflow rate distribution (red open) in our model and the percent particle collection (black open). ANOVA, analysis of variance; COPD, chronic obstructive pulmonary disorder.
In the same geometries created experimentally, CFPD modeling simulated particle transport and deposition to test the feasibility of the RTD lobe-specific targeting method and provide guidance to refine the experimental plan. The influence of geometry complexity, particle size, overall flow rate, and airflow distributions across the lobes on targeting was investigated. Figure 5 shows release maps for $1 \mu \mathrm{m}$ particles and $1 \mathrm{~L} / \mathrm{min}$ inlet flow rate inlets of geometries with three different upstream setups: the intubated model (Fig. 5A), the intubated model with an RTD nozzle (Fig. 5B), and a complete upper airway model (Fig. 5C). Particle release maps demonstrate that by controlling the particle deployment position and velocity, lobe-specific targeted drug delivery can be achieved in each of the geometries and experimental setups. As CFPD modeling was used to inspire the experimental plan, we first endeavored to closely recreate the proposed RTD setup within the CFPD simulations. With the presence of the RTD represented as a single inlet tube in the simulation, we find that the pulmonary airflow is locally disturbed at the entrance, resulting in different deposition sites (Fig. 5A, B). As observed in Figure 5A, the particles released inside the region denoted by a black circle will be $100 \%$ delivered to the LL lobe (marked as yellow). These released particles experience uniform inlet velocity, and no effect of the RTD device was considered in the simulations. In contrast, as observed in Figure $5 \mathrm{~B}$, the particles released from the same position inside the nozzle will be delivered to the left upper (LU)

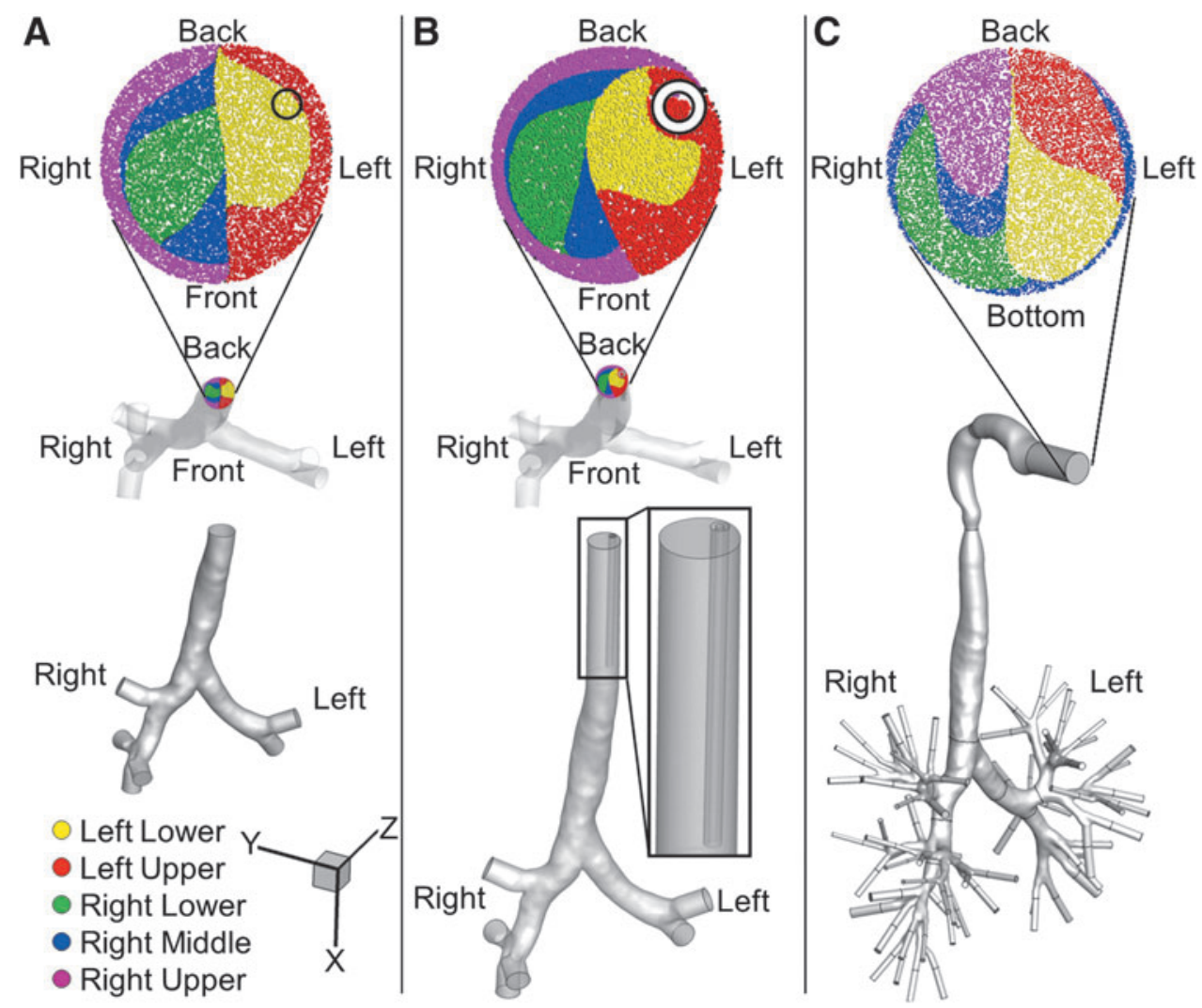

FIG. 5. CFPD geometries: representative CFPD results of inlet maps with different upstream setups: (A) intubated lung without an RTD, (B) intubated lung with an RTD, and (C) full throat model without an RTD. Release maps are all for $1 \mu \mathrm{m}$ particles and $1 \mathrm{~L} / \mathrm{min}$ inlet flow rate inlets. 
lobe (marked as red) due to the RTD effect. However, the presence of an RTD still results in inlet clustering and enables identification of entrance regions that will direct lobespecific deposition. Due to the RTD effects observed in the simulations, the in vitro results could deviate from the in silico results if the CFPD simulations did not include the RTD in the airflow domain. Due to the simplicity of the RTD-free CFPD approach, deposition maps determined in simulations without the RTD were initially used to inspire in vitro inlet location identification in both intubated and full airway geometries, with the acknowledgment that minor variations due to the nozzle effect will be expected.

In line with previous publications, ${ }^{(18,33)}$ lower inlet flow rates are preferred to generate more regulated laminar flow regimes and avoid turbulence due to the glottal contraction. These laminar regimes are predicted to improve the feasibility of directing the air-drug streams to a specific site with minimum disturbance by the airflow fluctuation (Fig. 5C). ${ }^{(18)}$ Due to this, the majority of the subsequent experiments were performed in a simplified intubated model (Fig. 6A and Supplementary Fig. S1), in line with simulations from Figure 5A and B. Experiments performed with the full lung model, as opposed to the intubated lung model, are explicitly noted throughout the article, whereas all other experiments are performed with the intubated model. With the higher inlet flow rate (e.g., $10 \mathrm{~L} / \mathrm{min}$ ) the available particle release region to target a specific lobe becomes smaller, indicating the enhanced difficulty to position the RTD (Fig. 6B). Thus, the preliminary numerical results elucidate that turbulence generated by the glottis will significantly increase the difficulty to target a specific lobe under a higher inlet flow rate. From computational results, it appears that targeted pulmonary delivery is still achievable at a higher flow rate $(10 \mathrm{~L} / \mathrm{min})$, without the upper airway and glottis geometry. In addition, it can be observed that particle size has a negligible effect on the particle release maps compared with the total flow rate (Fig. 6B). Finally, the flow rate distribution at the lobe outlet seems to have a significant effect on targeting locations, as can be seen by the comparison between healthy subject CFPD modeling and COPD patient CFPD modeling, where the airflow distribution between lobes shifts to a higher percentage of the upper lobes (Fig. 6C and Table 1). Although certain parameters, such as particle size, do not appear to affect the particle trajectories, it appears that flow rates, airflow profiles, and the presence of an RTD all influence particle trajectories and, thus, are predicted to affect regional targeting efficacy.

\section{Lobe-specific regional targeting}

After validating the experimental intubated model (Fig. 4), we sought to identify inlet locations that could yield lobe-specific particle collection. Inspired by CFPD simulations and using $1 \mu \mathrm{m}$ particles and an inlet flowrate of $1 \mathrm{~L} / \mathrm{min}$, the RTD was aligned at the inlet coordinates $\left(r=7.7 \mathrm{~mm}, \theta=36^{\circ}\right)$ (Supplementary Fig. S6). Under these conditions, we observed increased collection to the LL lobe totaling $86 \% \pm 6 \%$ of the overall fluorescent particle dose. As shown in Figure 7 , this corresponded to a $3 \times$ increase compared with the non-targeted collection of $29 \% \pm 4 \%$ $(p=0.0002)$. Collection in the right lobes also decreased significantly $(p<0.05$ for the right lower (RL), RM, and right upper (RU) lobes, additional statistics in Supplementary Fig. S7), indicating the potential to limit off-target collection. These results indicate the overall success of our RTD and demonstrate a general ability to localize aerosols to an individual lobe based solely on the inlet location.

Given the successful LL targeting, we explored additional inlet positions to identify ones that preferentially controlled collection to the remaining four lobes. Particle collection distributions from all five selected inlet locations demonstrating optimized collection profiles are shown in Figure 8. Overall, the percent particle collection for each lobe increased by at least $3 \times$ when compared with non-targeted collection, with at least $63 \%$ of the total particle collection directed to the desired lobe. The RTD locations targeting the LL, LU, and RU lobes ( $\left.r=6.2 \mathrm{~mm}, \theta=36^{\circ}\right],[r=7.7 \mathrm{~mm}$, $\left.\theta=324^{\circ}\right]$, and $\left.\left[r=4.7 \mathrm{~mm}, \theta=252^{\circ}\right]\right)$ increased particle collection to $86 \% \pm 6 \%, 92 \% \pm 9 \%$, and $90 \% \pm 5 \%$, respectively. Notably, these targeting locations also yielded less than $10 \%$ particle collection in the second highest lobe, demonstrating extremely efficient targeting locations. Slightly less efficient were inlet locations for the RM and RL lobes. The RTD location $\left(r=4.7 \mathrm{~mm}, \theta=180^{\circ}\right)$ increased particle collection in the RL lobe by $63 \% \pm 12 \%$, with $33 \%$ particle collection in the next highest lobe (RM). The RTD location

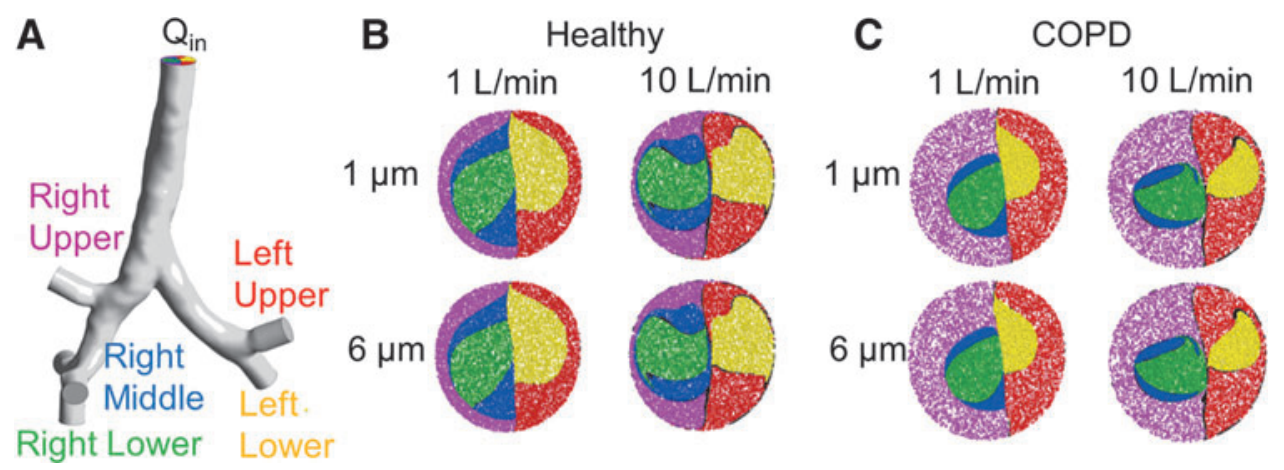

FIG. 6. CFPD particle sizes, flow rates, and airflow distributions: effects of particle size, total flow rate, and flow rate distribution across lobes on particle release map: (A) the simplified airway model and particle color legend, (B) particle release maps with a flow rate distribution representing a healthy subject varying particle size and flow rate, and (C) particle release maps with a flow rate distribution representing a COPD subject and varying particle size and flow rate. 

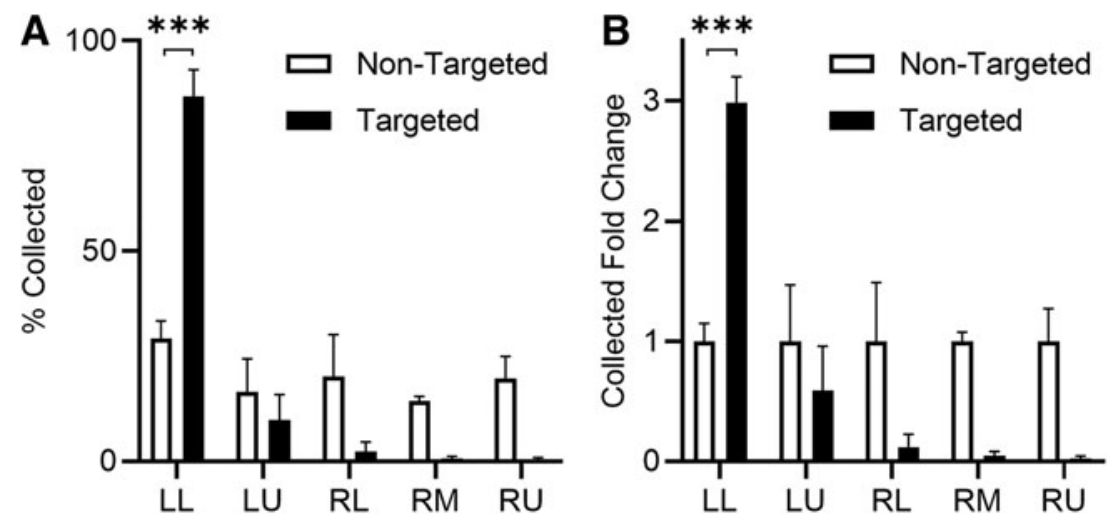

FIG. 7. LL lobe targeting. (A) Percent particle collection. The percent particle collection in each lobe in non-targeted and LL lobe targeted conditions in a healthy subject airflow distribution, with the targeted condition having the RTD at location $\left(r=7.7 \mathrm{~mm}, \theta=36^{\circ}\right)$. The non-targeted and targeted data were compared by using a two-way ANOVA, $n=3, p<0.0001$. The targeted and non-targeted percent collection in the lower left lobe was compared by using a Student's $T$-test, $n=3, p=0.0002$. (B) Collection fold change. The collection fold change in each lobe in non-targeted and LL lobe targeted conditions in a healthy subject airflow distribution, with the targeted condition having the RTD at location $\left(r=7.7 \mathrm{~mm}, \theta=36^{\circ}\right)$. The non-targeted and targeted data were compared by using a two-way ANOVA, $n=3, p<0.0001$. The targeted and non-targeted collection fold change in the lower left lobe was compared by using a Student's $T$-test, $n=3, p=0.0002$.

$\left(r=7.7 \mathrm{~mm}, \theta=180^{\circ}\right)$ increased particle collection in the RM lobe to $67 \% \pm 18 \%$, with $23 \%$ particle collection in the next highest lobe (RU).

In most instances, there was a direct correlation between where the CFPD modeling predicted particles would deposit and the resulting distribution of particles in the in vitro model. However, there were two distinct cases in which CFPD and in vitro results did not correlate, namely when targeting the RU and RM lobes. The RTD location of $\left(r=4.7 \mathrm{~mm}, \theta=252^{\circ}\right)$ demonstrated a significant increase in the RU lobe in vitro, however the CFPD model predicted that particles in this location would deposit into the RM lobe. Similarly, the RTD location $\left(r=7.7 \mathrm{~mm}, \theta=180^{\circ}\right)$ demonstrated a significant increase in the RM lobe in vitro whereas the CFPD model predicted that particles in this location would deposit into the RU lobe. However, inlet maps generated without considering the effects of the RTD were able to provide reasonable agreement with the majority of our experiments and were considered adequate for generating initial inlet locations to test. Detailed statistics on lobe deposition profiles compared against the non-targeted deposition profile are provided in Supplementary Figures S7 and S8 (fold change), with CFPD inlet maps showing the visual location of each targeting location. All locations attempted are shown in Supplementary Figure S9.

\section{Parameter variations: Particle size and inlet flow rate}

Particles of various sizes were used in place of the $1 \mu \mathrm{m}$ particles to determine whether particle size influences lobe targeting. Typically, particle size is known to affect the generation of deposition, such that particles between 1 and

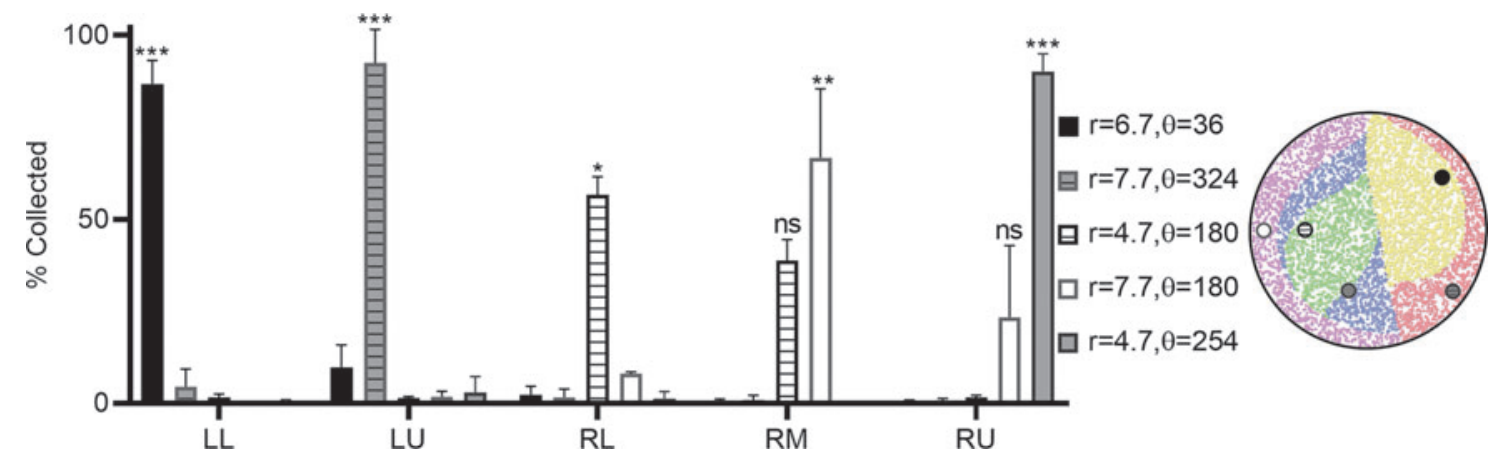

FIG. 8. RTD to all five lobes. Percent particle collection for each lobe when using five distinct targeting locations. Each lobe distribution was compared against the non-targeted data by using a two-way ANOVA, $n=3, p<0.0001$ for each profile. Student's $T$-tests, $n=3$, comparing the non-targeted collection with collection targeted to the LL, LU, RL, RM, and RU lobes, $p=0.0002,0.0003,0.015,0.008$, and 0.0005 , respectively. Inlet RTD locations are listed as well as overlaid on the CFPD map. LU, left upper; $\mathrm{RL}$, right lower; RM, right middle; RU, right upper. 
$10 \mu \mathrm{m}$ range in deposition from the alveolar region to the upper throat, respectively. ${ }^{(10)}$ Because of this, it is pharmaceutically relevant to consider particles in this range when determining the effects of particle size. Fluorescent and non-fluorescent polystyrene particles of 2.65 and $7.55 \mu \mathrm{m}$ were independently tested to determine the effects of larger particles on percent particle collection. The same location that had been previously used to target the LL lobe was used $\left(r=7.7 \mathrm{~mm}, \theta=36^{\circ}\right)$. As shown in Figure 9A, both the 2.65 and $7.55 \mu \mathrm{m}$ particles had significant collection in the LU lobe in addition to the LL lobe, and greater collection in the LU lobe than what had previously been seen when using $1 \mu \mathrm{m}$ particles at the same location. Specifically, the LL lobe increased to $86 \% \pm 6 \%$ with a $p$-value of 0.0002 for $1 \mu \mathrm{m}$ particles compared with non-targeted data; whereas the increase in collection is not statistically significant for 2.65 and $7.55 \mu \mathrm{m}$ particles when compared with nontargeted data, with increases to $39 \% \pm 9 \%$ and $40 \% \pm 17 \%$, respectively, $p>0.05$. Instead, particles seemed to preferentially collect in the LU lobe, with collection increasing to $60 \% \pm 14 \%$ and $45 \% \pm 9 \%$ for 2.65 and $7.55 \mu \mathrm{m}$ particles, respectively. The CFPD modeling of $1,2.65$, and $7.55 \mu \mathrm{m}$ did not predict the observed difference in targeting efficiency, as the inlet maps varied very little between particle sizes (Supplementary Fig. S10). However, as shown in Figure 9B, even with dispersion observed between the LL and LU lobes, more than $85 \%$ of the dose was distributed to the left lung by using the RTD, with particle collection increasing to $95 \% \pm 0.2 \%, 99 \% \pm 0.1 \%$, and $85 \% \pm 7 \%$ for 1 , 2.65 , and $7.55 \mu \mathrm{m}$ particles, respectively. This demonstrates considerable regional targeting at the lung level. Locations of the RTD and their comparison to CFPD model predictions are shown visually in Supplementary Figure S10.
To determine whether increasing the inlet flow rate from $1 \mathrm{~L} / \mathrm{min}$ toward more physiological flow rates would influence lobe-specific particle collection, higher flow rates were also evaluated for the same LL inlet position. The average inspiratory flow rate of an adult peaks between 30 and $60 \mathrm{~L} / \mathrm{min}^{(10)}$; however, preliminary CFPD modeling has shown that high flow rates increase mixing of particles (Fig. 6B). Due to CFPD predictions of flow rates that lead to mixing at the lower end of clinically relevant flow rates, ${ }^{(18)}$ inlet flow rates of 5 and $10 \mathrm{~L} / \mathrm{min}$ were used, maintaining the percent flow rate distribution to each lobe as what has been seen clinically (Supplementary Table S2). Particle collection was quantified in each lobe when using an RTD positioned at the same location that had previously been used to target the LL lobe $\left(r=7.7 \mathrm{~mm}, \theta=36^{\circ}\right)$. Increasing flow rate shifted collection to the LU lobe for both 5 and $10 \mathrm{~L} / \mathrm{min}$; specifically the LL collection for 1,5 , and $10 \mathrm{~L} / \mathrm{min}$ flow rates was $86 \% \pm 6 \%$, $62 \% \pm 12 \%$, and $61 \% \pm 12 \%$, respectively, whereas collection in the LU lobe was $10 \% \pm 6 \%, 38 \% \pm 13 \%$, and $37 \% \pm 13 \%$, respectively (Fig. 10A). However, the overall particle collection in the left lung was significantly higher than the right lung with increases to $95 \% \pm 0.02 \%, 99 \% \pm 0.7 \%$, and $98 \% \pm 1 \%$ for 1,5 , and $10 \mathrm{~L} / \mathrm{min}$ flow rate, respectively (Fig. 10B). This shift was aligned with CFPD modeling that shows a skewing of the definition of lobe boundaries at the inlet as flow rate increases (Fig. 6B). Locations of the RTD and their comparison to CFPD model predictions are shown visually in Supplementary Figure S10.

\section{COPD disease flow distribution}

We next evaluated RTD efficacy in changing air flow distributions at the lobe outlet. The same physical intubated
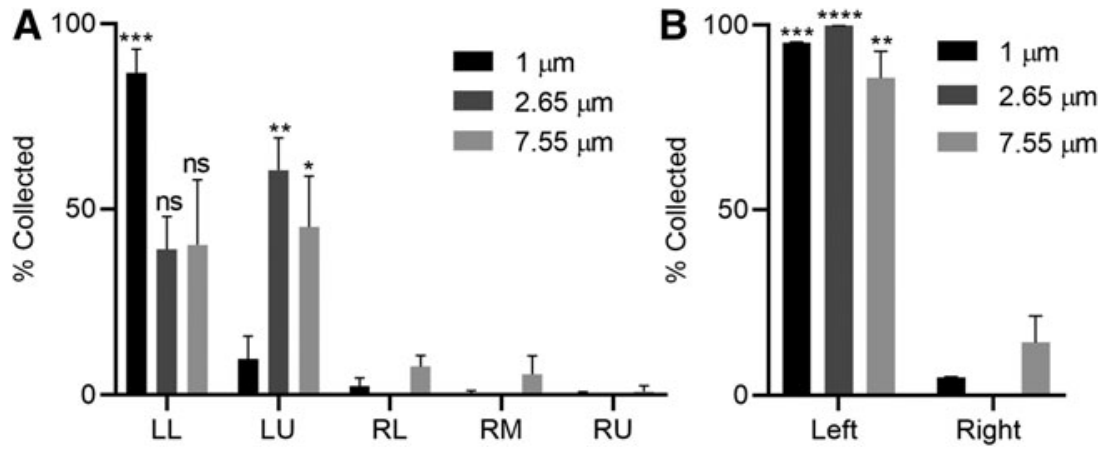

FIG. 9. Effect of particle size. The percent particle collection in each lobe for particles of $1,2.65$, and $7.55 \mu \mathrm{m}$ diameter particles when targeting location $\left(r=7.7 \mathrm{~mm}, \theta=36^{\circ}\right)$. (A) Each collection profile was compared with the nontargeted profile by using two-way ANOVA tests, $n=3, p<0.0001$ for 1 and $2.65 \mu \mathrm{m}$ particles and $p=0.0005$ for $7.55 \mu \mathrm{m}$ particles. Comparisons between the targeted and non-targeted LL lobe were performed by using Student's $T$-tests, $n=3, p=0.0002$ for $1 \mu \mathrm{m}$ particles and $p>0.05$ for 2.65 and $7.55 \mu \mathrm{m}$ particles. Comparisons between the targeted and non-targeted LU lobe were performed by using Student's $T$-tests, $n=3, p=0.002$ and 0.035 for 2.65 and $7.55 \mu \mathrm{m}$ diameter particles, respectively. (B) Left versus right profiles, which is the sum of the left lobes and right lobes collection, respectively. Each collection profile was compared with the non-targeted profile by using two-way ANOVA tests, $n=3, p<0.0001$ for all profiles. Comparison of the left lung collection was compared against the non-targeted collection by using Student's $T$-tests, $n=3, p=0.0009,0.00004$, and 0.0014 , for $1,2.65$, and $7.55 \mu \mathrm{m}$ particles, respectively. 

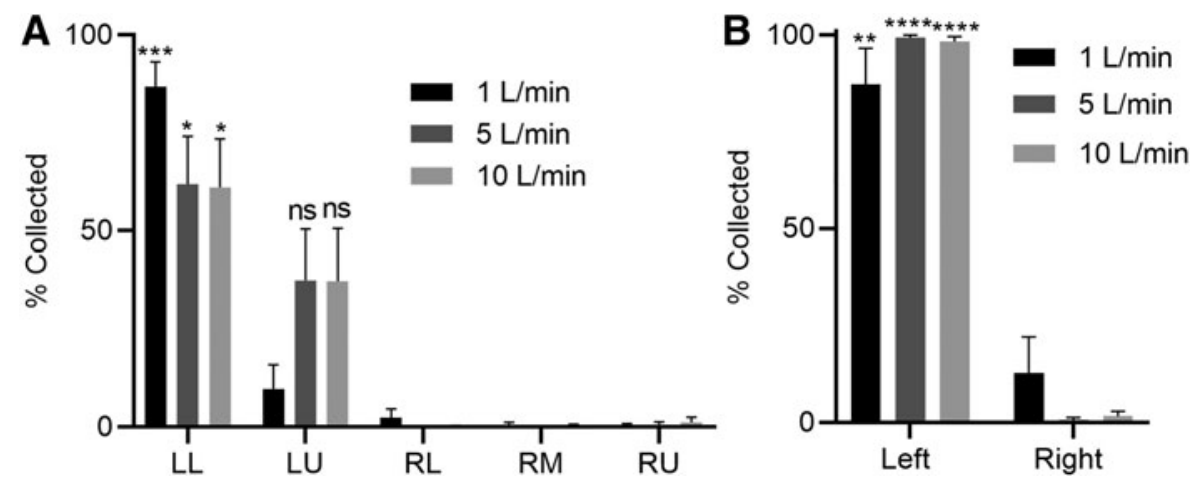

FIG. 10. Effect of inlet flow rate. The percent particle collection in each lobe for inlet flow rates of 1,5 , and $10 \mathrm{~L} / \mathrm{min}$ when targeting location $\left(r=7.7 \mathrm{~mm}, \theta=36^{\circ}\right)$. (A) Each collection profile was compared with the non-targeted profile by using twoway ANOVA tests, $n=3, p<0.0001$ for all profiles. Comparisons between the targeted and non-targeted LL lobe were performed by using Student's $T$-tests, $n=3$, $p=0.0002,0.012$, and 0.013 , for 1,5 , and $10 \mathrm{~L} / \mathrm{min}$, respectively. (B) Left versus right profiles, which is the sum of the left lobes and right lobes collection, respectively. Each collection profile was compared with the non-targeted profile by using two-way ANOVA tests, $n=3, p<0.0001$ for all profiles. Comparison of the left lung collection was compared against the non-targeted collection by using Student's $T$-tests, $n=3, p=0.0009,0.00005$, and 0.00006 for 1,5 , and $10 \mathrm{~L} / \mathrm{min}$, respectively.

replica was adapted by adjusting the lobe outlet flow rate distribution to match that of a patient with COPD. ${ }^{(32)}$ As shown in Table 1, the airflow distribution in COPD shifts to favor the upper lobes, with both RU and LU experiencing a greater proportion of the airflow. As with the previous airflow distribution, the percent airflow was compared against particle collection without the RTD; non-targeted particle collection is not statistically different than the set airflow distribution, suggesting that the COPD model accurately mimics clinically relevant particle collection and flow rates (Supplementary Fig. S11). In addition, a second condition was established by using airflow distributions between the reported healthy and diseased distribution to mimic disease progression, referred to as an "intermediate" model. Using the same inlet flow rates, CFPD modeling was performed to obtain particle inlet maps (Fig. 6C). The area of the inlet that could potentially target the RU and LU lobes was greater than compared with the map of a healthy adult, as was expected based on the increase in airflow to RU and LU. Using the in vitro model to quantify collection and the CFPD model to guide targeting locations, the LL lobe was targeted because this lobe experiences reduce airflow and, thus, a presumed reduction in therapeutic delivery. The same targeting location was used in the intermediate model, $\left(r=7.7 \mathrm{~mm}, \theta=36^{\circ}\right)$, as had previously been used when targeting the LL lobe in the healthy model. However, this location yielded almost entirely LU collection in the COPD model, as was predicted with CFPD modeling, and thus other locations were tried in addition that were predicted to deposit in the LL lobe based on CFPD modeling. When targeting in the intermediate model, there was an increase of $35 \% \pm 5 \%$ in particle collection in the LL lobe $(p=0.0015)$, a $3 \times$ increase compared with the non-targeted collection (Fig. 11). When targeting in the COPD model at location $\left(r=2.7 \mathrm{~mm}, \theta=36^{\circ}\right)$, the collection in the LL lobe was $16 \% \pm 5 \%$ and this increase was not statistically significantly different from the non-targeted collection. However, the LU lobe collection increased to $69 \% \pm 1 \%$ when targeting in the
COPD model $(p=0.002)$ a $1.5 \times$ increase compared with the non-targeted collection. As the severity of COPD increased, and thus decreased the airflow distribution to the LL, the particle collection in the targeted LL lobe decreased whereas the collection in LU increased, most likely due to the increased airflow.

\section{Model complexity}

Our previous experiments considered lobe-specific targeting below the glottis by using the intubated 3D geometry, as CFPD modeling has demonstrated the potential for turbulent flow and mixing in this area (Fig. 5). However, we desired to apply our RTD in a more complex model that includes the throat to determine whether lobe-specific targeting via controlled inlet location could be adapted to an inhaler design. We hypothesized that lung targeting (i.e., increasing collection in the left or right lung when targeted) would be possible, even if lobe-specific targeting would not be possible. In the full throat geometry using the healthy airflow distribution from Table 1, a mouth inlet location of $\left(r=7.7 \mathrm{~mm}, \theta=0^{\circ}\right)$ increased collection in the left lung to $87 \% \pm 9 \% \quad(p=0.002)$, corresponding to a $1.9 \times$ increase over the non-targeted collection (Fig. 12). This demonstrated the ability to target the left or right lung significantly by using the mouth inlet location. Within these same data, we observed that the LU lobe particle collection increased to $65 \% \pm 4 \%(p=0.005)$, a $4 \times$ increase compared with the non-targeted collection. The second highest lobe of percent particle collection was the LL lobe with $23 \%$, which was not statistically significantly different compared with the nontargeted collection. This suggests that lobe-specific particle collection is, in fact, possible by simply controlling the mouth inlet location, even in a complex model containing the glottis constriction.

In addition to the left lung targeting, right lung targeting was performed by using location $\left(r=7.7 \mathrm{~mm}, \theta=180^{\circ}\right)$. Percent particle collection the right lung increased to 

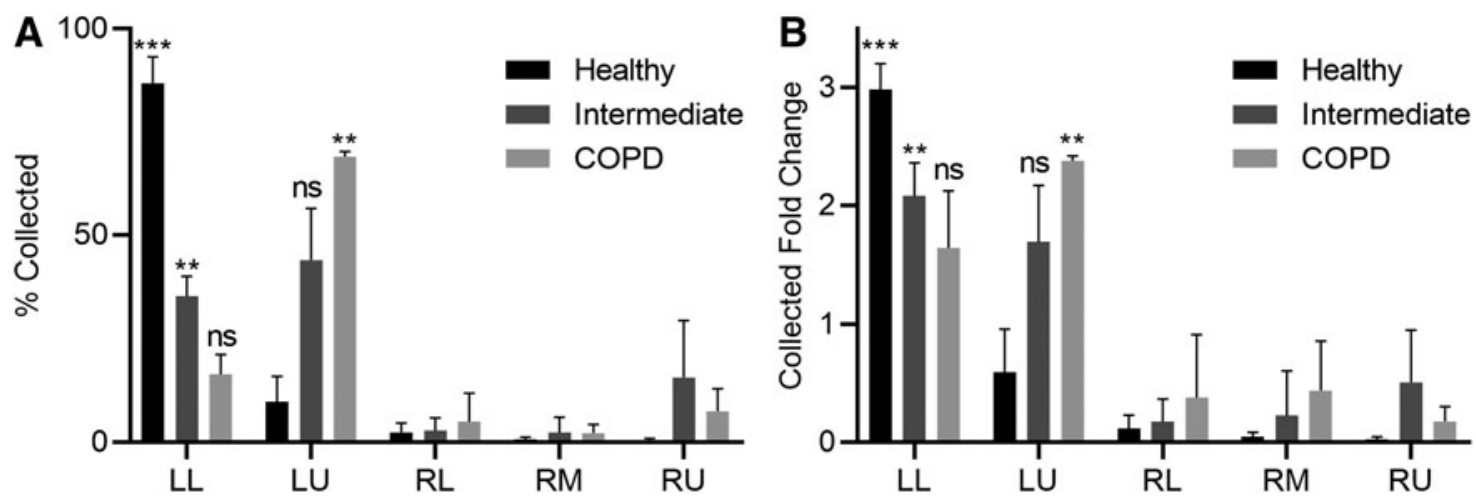

FIG. 11. Effect of background airflow distribution: COPD model. (A) Percent particle collection. Targeting of the LL lobe in the intermediate and COPD models compared with that of a healthy subject model. Targeting location used for the healthy and intermediate data was $\left(r=7.7 \mathrm{~mm}, \theta=36^{\circ}\right)$ and was $\left(r=2.7 \mathrm{~mm}, \theta=36^{\circ}\right)$ for the COPD data. Comparison between the healthy targeted data and the healthy non-targeted data was performed by using a two-way ANOVA test, $n=3, p<0.0001$. Comparison between both the intermediate and COPD targeted LL lobe collection against the COPD non-targeted LL lobe collection was performed by using Student's $T$-tests, $n=3, p=0.001$ and 0.23 for the intermediate and COPD models, respectively. Comparison between both the intermediate and COPD targeted LU lobe collection against the COPD non-targeted LU lobe collection was performed by using Student's $T$-tests, $n=3, p=0.1$ and 0.002 for the intermediate and COPD models, respectively. (B) Collection fold change. Targeting of the LL lobe in the intermediate and COPD models compared with that of a healthy subject model. Targeting location used for the healthy and intermediate data was $\left(r=7.7 \mathrm{~mm}, \theta=36^{\circ}\right)$ and was $\left(r=2.7 \mathrm{~mm}, \theta=36^{\circ}\right)$ for the COPD data. Comparison between the healthy targeted data and the healthy non-targeted data was performed by using a two-way ANOVA test, $n=3, p<0.0001$. Comparison between both the intermediate and COPD targeted LL lobe fold change against the COPD non-targeted LL lobe fold change was performed by using Student's $T$-tests, $n=3, p=0.001$ and 0.23 for the intermediate and COPD models, respectively. Comparison between both the intermediate and COPD targeted LU lobe fold change against the COPD non-targeted LU lobe fold change was performed by using Student's $T$-tests, $n=3, p=0.1$ and 0.002 for the intermediate and COPD models, respectively.
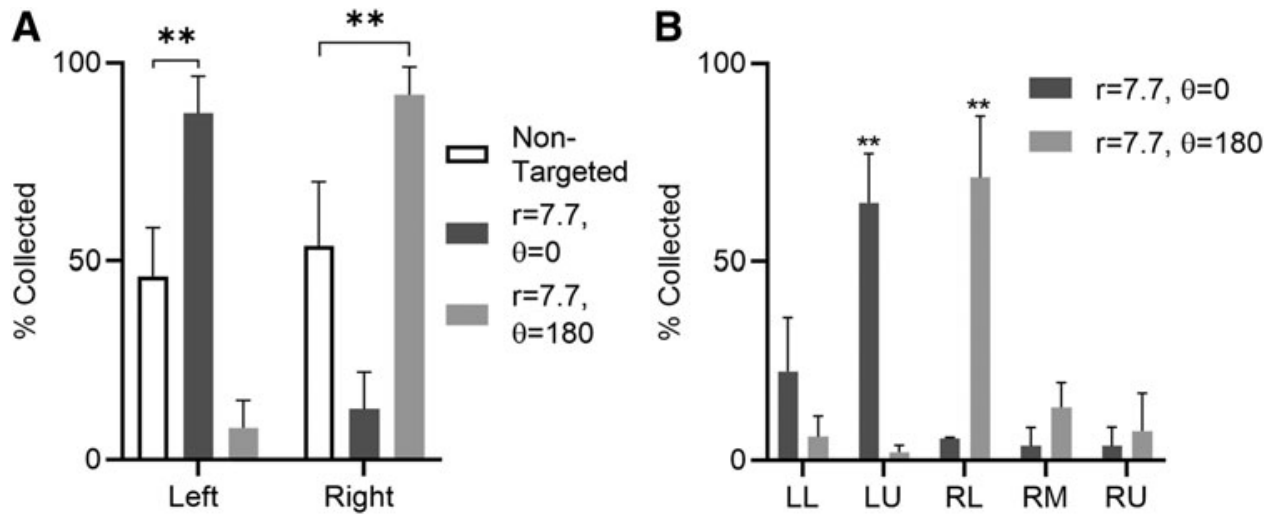

FIG. 12. RTD in model with upper throat. Targeting in a model that includes the upper throat showing significant efficiency at targeting the left $\left(r=7.7 \mathrm{~mm}, \theta=0^{\circ}\right)$ and right $\left(r=7.7 \mathrm{~mm}, \theta=180^{\circ}\right)$ lungs. (A) Left versus right lung collection, which is the sum of the left lobes and right lobes collection, respectively. Comparison between targeted and nontargeted collection profiles was compared by using two-way ANOVA tests, $n=3$, $p<0.0001$. Comparison between targeted and non-targeted collection in each lung was performed by using Student's $T$-tests, $n=3, p=0.002$ and 0.0016 for the left and right lung, respectively. (B) The lobe-specific distribution. Comparison between the targeted and nontargeted collection profiles was compared by using two-way ANOVA tests, $n=3$, $p<0.0001$. Comparison between the targeted and non-targeted lobe collection was performed by using Student's $T$-tests, $n=3, p=0.005$ and 0.008 for the LU and RL lobes, respectively. 
$91 \% \pm 7 \%(p=0.0016)$, a $1.7 \times$ increase over non-targeted data (Fig. 12). The lobe-specific analysis demonstrated $71 \% \pm 16 \%$ collection in the RL lobe $(p=0.008)$, a $3.5 \times$ increase compared with non-targeted data. The second highest lobes of collection were the RM and RU lobes, having $13 \%$ and $7 \%$ of the total percent particle collection, respectively; this was found not to be statistically different from the non-targeted collection. Compared with the intubated model, the full throat model had $27 \%$ less particle collection in the LU lobe and $8 \%$ less particle collection in the left lung. In addition, the full throat model had $8 \%$ more particle collection in the RL lobe, but $6 \%$ less particle collection in the right lung compared with the intubated model.

\section{Discussion}

Realization of lobe-specific aerosol delivery via inhalation would offer development for novel treatment paradigms for severe pulmonary conditions that have lobe-specific effects. To experimentally establish the feasibility of leveraging aerosol inlet location to alter the lobe-specific distribution of deposited aerosols in the lung, we have evaluated an RTD in an anatomically relevant 3D printed lung model with physiological lobe-outlet flow distributions. Our approach mirrors those from other mouth-throat lung phantom models made with rapid prototyping methods, studying deposition after a single inhalation. ${ }^{(22-25)}$ Mouth throat models such as this have been successful in predicting clinical deposition when paired with a breath simulator. ${ }^{(34,35)}$ With our approach, we demonstrate that regional distribution can be efficiently achieved to the right and left lung with the RTD aligned at the mouth inlet, whereas lobe-specific distribution can be highly efficient for all five human lobes when the RTD is aligned at an intubated outlet position. Overall targeting efficiency was found to require precision alignment between the inlet position and airway geometry and was highly dependent on the inlet position, particle size, inlet flow rate, and background airflow distribution.

The majority of our experiments were performed in the truncated intubated model, omitting the upper extrathoracic airway and bypassing potential turbulence within the trachea. ${ }^{(18)}$ Evaluating the RTD in this manner enabled us to identify inlet locations for each of the five individual lobes and demonstrate proof-of-concept for regional lobe-specific aerosol delivery. While providing advantages for this initial evaluation, truncating the model in such a manner alters the background airflow distribution; in a dynamic human larynx, glottis geometry generates a complicated threedimensional focusing jet ${ }^{(36-38)}$ that is further altered temporally by the glottal expansion and contraction during respiration, ${ }^{(39)}$ all of which is known to impact downstream aerosol deposition and is not present in our static 3D-printed intubated model. ${ }^{(32,36-39)}$ Similar proof-of-concept simplifications were made by using the fully developed flow assumption in our CFPD models, which does not capture the known recirculation pattern at the inlet of the trachea. ${ }^{(40)}$ Further, our experimental model assessed only a single inhalation, as opposed to a periodic respiratory cycle, and was evaluated at low overall flow rates and collection periods of 10 seconds to enable adequate particle deposition at the outlet. Collectively, our simplifications enabled scenarios that mimic the lobe outlet flow distribution and the depth an aerosol might be released from an endotracheal tube within a relevant geometric structure; however, our setup does not completely replicate the full complexity of cyclical respiratory flow. As a result, implementation of a modified RTD in vivo will likely experience varied airflow conditions from those presented here and will require further assessment in increasingly complicated environments. This limitation of the intubated model prompted our evaluation of regional targeting from the mouth inlet in a complete mouth throat model (Fig. 12). Although these data showed reduced efficiency in lobe-specific targeting compared with the intubated model, there was still a significant increase in lobe collection and, with further optimization, lobe-specific targeting from an inhaler with coordinated timing release could be possible. Experiments including the upper airways do, however, demonstrate efficient lung-specific targeting, suggesting that a minimum of lung-specific targeting may be achievable with an inhaler and controlled release positions at the mouth. In addition, these experiments suggest that at higher flow rates, effects from turbulence in models that include the upper airways will reduce the feasibility of lobespecific targeting such that only lung-specific targeting would be expected. Future work will involve further exploration of mouth inlet positions linked to personalized anatomies, as well as the development of a modified RTD within an endotracheal tube to assess regional targeting in the presence of a more realistic airflow profile from the upper airways.

Our results point to a number of potential challenges toward the overall goal of realizing lobe-specific targeting. Interestingly, the ability to target specific lobes varied among the five and was unsurprisingly impacted by variations in the lobe outlet flow distribution, as would be present in certain disease conditions. Although all five lobes were successfully targeted with the $1 \mu \mathrm{m}$ particles at $1 \mathrm{~L} / \mathrm{min}$ inlet flow, identification of efficient locations for the RM and RL lobes was less efficient than the LL, LU, and RU lobes (Fig. 8). Further, the final experimental inlet locations identified for the RM and RU lobes were the only two positions that did not fully agree with CFPD predictions. The final RU RTD location $\left(r=4.7 \mathrm{~mm}, \theta=252^{\circ}\right)$ was predicted via CFPD to deposit into the RM lobe, whereas the final $R M$ RTD location $\left(r=7.7 \mathrm{~mm}, \theta=180^{\circ}\right)$ was predicted via CFPD to deposit into the RU lobe. Both of the final experimental locations resulted in collection split across two distinct lobes, with the desired lobe having significantly more collection than the untargeted collection. Identification of inlet positions that selectively target either the RM or RL lobe may have been challenging to optimize in our current experimental setup due to our chosen alignment approach for the RTD device design. These two lobes also appear to have the most complicated and smallest inlet areas according to the CFPD release maps and suggest a high degree of sensitivity around the precise location to successfully achieve targeting to these lobes. Alignment of the model with gravity may further influence lobe-specific delivery. ${ }^{(15,16)}$ The reported discrepancy between these experimental cases and CFPD is most likely due to the issues previously discussed, with the RTD generating a nozzle that influences the prediction of lobe deposition. Certainly, 
additional inlet locations may yield further increases to these two lobes, as we are far from exhausting the entire inlet parameter space. However, our overall data demonstrate that it is possible to at least preferentially skew percent particle collection to all five lobes within a healthy airflow distribution.

Our work also points to challenges in implementing lobespecific targeting as the lobe outlet distribution varies. As was seen in our work, diseased air profiles are expected to play a critical role in the progression of lobe-specific targeting. In the COPD-patient inspired airflow profile, we assessed multiple inlet locations to achieve increased delivery to our target in the LL (Fig. 11). Although we were unable to find a location able to increase LL in the reported COPD profile, we were able to demonstrate a significantly increased targeting in the intermediate condition. It could be possible to yet achieve higher efficacy in targeting the LL lobe through additional evaluation of inlet locations; however, these results indicate the challenges in lobe-specific targeting as airflow distributions to an individual lobe decrease. Thus, it may be possible to employ lobe-specific targeting to a lobe with decreased airflow in a mild disease state but not in a severe state. Further assessments of the other four lobes in decreasing flow distributions are needed to fully characterize this effect throughout the lung. Specific aspects of disease progression and background flow profiles will likely play a significant role in targeting, and it is possible that certain disease states with restricted airflow to the disease location will never be amenable to this approach. Thus, implementation of lobe-specific targeting will not only require customization to the overall inlet flow rates and patient-specific anatomy but lobe-specific outlet flow distributions as well. Likely, consideration of lower airways and cyclical breathing profiles in future in vitro models will provide further increased airflow mimicry to our model and enable predictions of actual deposition within the lobe, as these would be expected to also influence the local airflow. ${ }^{(41)}$ A cyclic breathing profile would also elucidate exhalation of particles and allow for an accurate quantification of delivered dose. In addition, generating lung replicas from a population of patients with a range of severity of COPD would improve the evaluation of this technique in a disease environment, as this replica was based on CT scans of a healthy subject. Further, inclusion of lower airways would enable generation-level quantification of deposition within each lobe, rather than merely aerosols entering the lobe. Thus, our data further suggest the need for advanced airflow understanding in relevant disease models to realize regional targeting in the clinic, as well as highlighting advances needed in subsequent in vitro models.

Finally, our data point to challenges faced by future pharmaceutical formulations developed for this technique. Regionally delivered aerosols will require attention to the precise particle size and device flow rates, as both will impact the efficiency of delivery and efficacy of the inhalable therapeutic. Our results of varied particle sizes (Fig. 9) demonstrate that a targeting strategy for $1 \mu \mathrm{m}$ particles does not translate directly to larger particle sizes. Thus, strategies tailored to narrow particle size distributions would be required. Uniquely from the CPFD results, our experimental results further suggest that the process of identifying a location for lobe-specific targeting could be specific to parti- cle size, requiring optimization for each particle diameter. Notably, particles evaluated in this study were model systems, yielding highly monodisperse aerosols from uniform spherical particles of a limited size range. These are not representative of most pharmaceutical powders. ${ }^{(1)}$ Monodisperse aerosols needed for successful lobe-specific regional delivery may be feasible by using atomization techniques that yield homogeneous droplet sizes, ${ }^{(42,43)}$ or through nano- and micro-molding techniques that are capable of generating particles of narrow dispersity. ${ }^{(44,45)}$ The ability to achieve a target dose requirement within the regionally targeted lobe remains to be determined, as this approach is applied to drug-carrying pharmaceutical powders and likely requires delivery over repeated breathing cycles due to the dilute aerosol concentrations delivered here. Certainly, the low operational flow rates demonstrated by our work (Figs. 6 and 10) and others ${ }^{(18,33)}$ to enable lobe-specific regional targeting pose considerable challenges to implementation in a physiologically relevant lung environment.

For regional lobe-specific targeting as proposed to be realized clinically, considerably more preclinical work is needed to overcome these challenges. The demonstrated 3Dprinted lung model captures only limited aspects of the human airways, pointing to a need to advance in vitro lung models that are capable of assessing lobe-specific regional delivery. Leveraging benefits and recent advances in 3D printing, evaluation of particle deposition in such in vitro tools will continue to expand the scope of quantifiable data in aerosol research, enabling assessments of a wider range of patient geometries needed to generalize this approach. In addition, modifications such as slow breathing patterns or the use of low-density gases could be evaluated to implement regional delivery by avoiding limitations demonstrated to be caused by turbulence. Future work in elastic and mucus-mimicking lung models that include both upper and peripheral airways will overcome limitations of the existing model and increase the translational potential of our results.

Despite the challenges and remaining obstacles, we are optimistic for the implementation of lobe-specific regional targeting informed by patients' CT scans to allow for personalized medicine and targeted delivery. Our results suggest that highly efficient lobe-specific delivery will likely require inlet positions below the trachea. This may be realized through development of an endotracheal tube or bronchoscope adaptor, building on controlled release positions identified in this work. In addition, clinically relevant therapeutics, disease models, and synchronized aerosol release will need to be evaluated in tandem to determine drug dosing effects and potential therapeutic benefits of lobespecific regional targeting.

\section{Conclusion}

We have successfully developed an in vitro model to quantify lobe targeting in a 3D-printed lung replica and demonstrate the utility of a novel RTD to control the inlet position of fluorescent polystyrene particles. Together, this model and device were able to realize lobe-specific particle collection such that up to $92 \%$ of fluorescent particles entering the model were collected in a targeted lobe. We demonstrate for the first time experimentally the ability to 
target particle collection to an individual lobe, which would reduce off-target effects and improve the delivery of inhaled therapeutics. Although our results demonstrate the necessity for narrow control over particle size, inlet flow rate, and lobe airflow distributions, this work represents a small step forward in realizing lobe-specific regional targeting. It is our hope that this work will create opportunities for future work in regional targeting, leading to innovation in aerosol therapeutic delivery and improved treatments for patients.

\section{Acknowledgments}

The authors acknowledge Ian Woodward and Emma Peterman for their helpful discussions.

\section{Author Disclosure Statement}

The authors declare they have no competing financial interests.

\section{Funding Information}

This work was supported by internal University of Delaware funds received by C.A.F.

\section{Supplementary Material}

Supplementary Data

Supplementary Figure S1

Supplementary Figure S2

Supplementary Figure S3

Supplementary Figure S4

Supplementary Figure S5

Supplementary Figure S6

Supplementary Figure S7

Supplementary Figure S8

Supplementary Figure S9

Supplementary Figure S10

Supplementary Figure S11

Supplementary Table S1

Supplementary Table S2

\section{References}

1. Rau JL: The inhalation of drugs: Advantages and problems. Respir Care. 2004;50:367-382.

2. Kim SS, Seo JB, Lee HY, Nevrekar DV, Forssen AV, Crapo JD, Schroeder JD, and Lynch DA: Chronic obstructive pulmonary disease: Lobe-based visual assessment of volumetric CT by using standard images-Comparison with quantitative CT and pulmonary function test in the COPDGene Study. Radiology. 2013;266:626-635.

3. Cameron JL, Mitchell WH, and Zuidema GD: Aspiration pneumonia: Clinical outcome following documented aspiration. Arch Surg. 1973;106:49-52.

4. Byers TE, Vena JE, and Rzepka TF: Predilection of lung cancer for the upper lobes: An epidemiologic inquiry. J Natl Cancer Inst. 1984;72:1271-1275.

5. Otterson GA, Villalona-Calero MA, Sharma S, Kris MG, Imondi A, Gerber M, White DA, Ratain MJ, Schiller JH, Sandler A, Kraut M, Mani S, Murren JR: Phase I Study of inhaled doxorubicin for patients with metastatic tumors to the lungs. Clin Cancer Res. 2007;13:1246-1252.

6. Sul B, Wallqvist A, Morris MJ, Reifman J, and Rakesh V: A Computational study of the respiratory airflow charac- teristics in normal and obstructed human airways. Comput Biol Med. 2014;52:130-143.

7. Ernst A, Feller-Kopman D, Becker HD, and Mehta AC: Central airway obstruction. Am J Respir Crit Care Med. 2004;169:1278-1297.

8. Patton JS, Brain JD, Davies LA, Fiegel J, Gumbleton M, Kim K-J, Sakagami M, Vanbever R, and Ehrhardt C: The particle has landed-Characterizing the fate of inhaled pharmaceuticals. J Aerosol Med Pulm Drug Deliv. 2010; 23(S2):S-71-S-87.

9. Otterson GA, Villalona-Calero MA, Hicks W, Pan X, Ellerton JA, Gettinger SN, and Murren JR: Phase I/II Study of inhaled doxorubicin combined with platinum-based therapy for advanced non-small cell lung cancer. Clin Cancer Res. 2010;16:2466-2473.

10. Patton JS, and Byron PR: Inhaling medicines: Delivering drugs to the body through the lungs. Nat Rev Drug Discov. 2007;6:67-74.

11. Darquenne C, Fleming JS, Katz I, Martin AR, Schroeter J, Usmani OS, Venegas J, and Schmid O: Bridging the gap between science and clinical efficacy: Physiology, imaging, and modeling of aerosols in the lung. J Aerosol Med Pulm Drug Deliv. 2016;29:107-126.

12. Dunnill MS: The pathology of asthma, with special reference to changes in the bronchial mucosa. J Clin Pathol. 1960;13, 27-33.

13. Sznitman J: Respiratory microflows in the pulmonary acinus. J Biomech. 2013;46:284-298.

14. Plank C: Nanomagnetosols: Magnetism opens up new perspectives for targeted aerosol delivery to the lung. Trends Biotechnol. 2008;26:59-63.

15. Oakes JM, Marsden AL, Grandmont C, Shadden SC, Darquenne C, and Vignon-Clementel IE: Airflow and particle deposition simulations in health and emphysema: From in vivo to in silico animal experiments. Ann Biomed Eng. 2014;42:899-914.

16. Bennett WD, Brown JS, Zeman KL, Hu S, Scheuch G, and Sommerer K: Targeting delivery of aerosols to different lung regions. J Aerosol Med. 2002;15179-188.

17. Celikoglu F, Celikoglu SI, and Goldberg EP: Techniques for intratumoral chemotherapy of lung cancer by bronchoscopic drug delivery. Cancer Ther. 2008;6:545-552.

18. Feng Y, Chen X, and Yang M: An in silico investigation of a lobe-specific targeted pulmonary drug delivery method. In Engineers, A. S. of M., Ed.; 2018 Design of Medican Devices Conference; pp. V001T08A011-V001T08A011, 2018.

19. Kleinstreuer C, Zhang Z, Li Z, Roberts WL, and Rojas C: A new methodology for targeting drug-aerosols in the human respiratory system. Int J Heat Mass Transf. 2008;51:55785589.

20. Kleinstreuer C, and Seelecke S: Inhaler system for targeted maximum drug-aerosol delivery. 7,900,625, March 2011.

21. Marple VA, Roberts DL, Romay FJ, Miller NC, Truman KG, Van Oort M, Olsson B, Holroyd MJ, Mitchell JP, and Hochrainer D: Next generation pharmaceutical impactor (A new impactor for pharmaceutical inhaler testing). Part I: Design. J Aerosol Med. 2003;16:283-299.

22. Wei X, Hindle M, Delvadia RR, and Byron PR: In vitro tests for aerosol deposition. V: Using realistic testing to estimate variations in aerosol properties at the trachea. J Aerosol Med Pulm Drug Deliv. 2017;30:339-348.

23. Huynh BK, Traini D, Farkas DR, Longest PW, Hindle M, and Young PM: The development and validation of an 
in vitro airway model to assess realistic airway deposition and drug permeation behavior of orally inhaled products across synthetic membranes. J Aerosol Med Pulm Drug Deliv. 2017;30:1-6.

24. Nordlund M, Belka M, Kuczaj AK, Lizal F, Jedelsky J, Elcner J, Jicha M, Sauser Y, Le Bouhellec S, Cosandey S, Majeed S, Vuillaume G, Peitsch MC, Hoeng J.: Multicomponent aerosol particle deposition in a realistic cast of the human upper respiratory tract. Inhal Toxicol. 2017;29: 113-125.

25. Lizal F, Elcner J, Hopke PK, Jedelsky J, and Jicha M: Development of a realistic human airway model. Proc Inst Mech Eng Part H J Eng Med. 2012;226:197-207.

26. Feng Y, Zhao J, Chen X, and Lin J: An in silico subjectvariability study of upper airway morphological influence on the airflow regime in a tracheobronchial tree. Bioengineering. 2017;4:90-103.

27. Menter FR, Langtry R, and Völker S: Transition modelling for general purpose CFD codes. Flow Turbul Combust. 2006;77:277-303.

28. Menter FR: Two-equation Eddy-viscosity turbulence models for engineering applications. AIAA J. 1994;32: 1598-1605.

29. Chen X, Feng Y, Zhong W, and Kleinstreuer C: Numerical investigation of the interaction, transport and deposition of multicomponent droplets in a Simple Mouth-Throat Model. J Aerosol Sci. 2017;105:108-127.

30. Li A, and Ahmadi G: Deposition of aerosols on surfaces in a turbulent channel flow. Int J Eng Sci. 1993;31:435-451.

31. Saffman PG: The lift on a small sphere in a slow shear flow. J Fluid Mech. 1965;22:385-400.

32. Sul B, Oppito Z, Jayasekera S, Vanger B, Zeller A, Morris M, Ruppert K, Altes T, Rakesh V, Day S, Robinson R, Reifman J, Wallqvist A : Assessing airflow sensitivity to healthy and diseased lung conditions in a Computational Fluid Dynamics Model validated in vitro. J Biomech Eng. 2018;140:051009-1-05009-05014.

33. Nicolaou L: Inertial and gravitational effects on aerosol deposition in the conducting airways. J Aerosol Sci. 2018; 120:32-51.

34. Delvadia RR, Longest PW, and Byron PR: In vitro tests for aerosol deposition. I: Scaling a physical model of the upper airways to predict drug deposition variation in normal humans. J Aerosol Med Pulm Drug Deliv. 2012; 25:32-40.

35. Golshahi L, Noga ML,Vehring R, and Finlay WH: An in vitro study on the deposition of micrometer-sized particles in the extrathoracic airways of adults during tidal oral breathing. Ann Biomed Eng. 2013;41:979-989.

36. Zheng X, Mittal R, and Xue Q: Direct-numerical simulation of the glottal jet and vocal-fold dynamics in a three- dimensional laryngeal model. J Acoust Soc Am. 2011;130: 404-415.

37. Xi J, Longest PW, and Martonen TB: Effects of the laryngeal jet on nano- and microparticle transport and deposition in an approximate model of the upper tracheobronchial airways. J Appl Physiol. 2008;104:1761-1777.

38. Corcoran TE, and Chigier N: Characterization of the laryngeal jet using phase doppler interferometry. J Aerosol Med. 2000;13:125-127.

39. Zhao J, Feng Y, and Fromen CA: Glottis motion effects on the particle transport and deposition in a subject-specific mouth-to-trachea model: A CFPD study. Comput Biol Med. 2020;116 103532.

40. Oakes JM, Roth SC, and Shadden SC: Airflow simulations in infant, child, and adult pulmonary conducting airways. Ann Biomed Eng. 2018;46, 498-512.

41. Longest WP, and Vinchurkar S: Validating CFD predictions of respiratory aerosol deposition: Effects of upstream transition and turbulence. J Biomech. 2007;40:305-316.

42. Ijsebaert SJC, Geerse KB, Marijnissen JCM, Lammers JJ, and Zanen P: Electro-hydrodynamic atomization of drug solutions for inhalation purposes. J Appl Physiol. 2001;91: 2735-2741.

43. Alvarez M, Friend J, and Yeo LY: Rapid generation of protein aerosols and nanoparticles via surface acoustic wave atomization. Nanotechnology. 2008;19:455103.

44. Fromen CA, Shen TW, Larus AE, Mack P, Maynor BW, Luft JC, and DeSimone JM: Synthesis and characterization of monodisperse uniformly shaped respirable aerosols. AIChE J. 2013;59:3184-3194.

45. Garcia A, Mack P, Williams S, Fromen CA, Shen TW, Tully J, Pillai J, Kuehl P, Napier ME, DeSimone JM, and Maynor BW: Microfabricated engineered particle systems for respiratory drug delivery and other pharmaceutical applications. J Drug Deliv. 2012;2012:941243.

Received on August 9, 2019 in final form, June 14, 2020

Reviewed by: Jessica Oakes

Address correspondence to: Catherine A. Fromen, PhD

Department of Chemical and Biomolecular Engineering University of Delaware, Newark 150 Academy Street Newark, DE 19716 USA

E-mail: cfromen@udel.edu 